\title{
Enhanced sensitivity to the fine-structure constant variation in Th IV atomic clock transition
}

\author{
V. V. Flambaum ${ }^{1,2}$ and S. G. Porsev ${ }^{1,3}$ \\ 1 School of Physics, University of New South Wales, Sydney, NSW 2052, Australia \\ 2 New Zealand Institute for Advanced Study, Massey University (Albany Campus), \\ Private Bag 102904, North Shore MSC Auckland, New Zealand and \\ 3 Petersburg Nuclear Physics Institute, Gatchina, Leningrad district, 188300, Russia
}

(Dated: November 20, 2018)

\begin{abstract}
Our calculations have shown that the $5 f_{5 / 2}-7 s_{1 / 2} 23131 \mathrm{~cm}^{-1}$ transition from the ground state in the ion $\mathrm{Th}^{3+}$ is very sensitive to the temporal variation of the fine structure constant $\alpha=e^{2} / \hbar c$ $\left(q=-75300 \mathrm{~cm}^{-1}\right)$. The line is very narrow, the ion has been trapped and laser cooled and the positive shifter line $5 f_{5 / 2}-5 f_{7 / 2} 4325 \mathrm{~cm}^{-1}\left(q=+2900 \mathrm{~cm}^{-1}\right)$ may be used as a reference. A comparison may also be made with a positive shifter in another atom or ion. This makes $\mathrm{Th}^{3+} \mathrm{a}$ good candidate to search for the $\alpha$ variation.

PACS numbers: 31.30.Gs, 06.20.Jr, 31.15.am
\end{abstract}

\section{INTRODUCTION}

Theories unifying gravity with other interactions suggest temporal and spatial variation of the fundamental "constants" in expanding Universe (see e.g. review [1]). The spatial variation can explain fine tuning of the fundamental constants which allows humans (and any life) to appear. We appeared in the area of the Universe where the values of the fundamental constants are consistent with our existence. The fundamental constants may be slightly different near massive bodies (see e.g. review [2]). There are some hints for the variation of different fundamental constants in quasar absorption spectra [3, 4, ㅎ, [6, 7, 8] and Big Bang nucleosynthesis [9, 10] data. However, a majority of publications report limits on the variations of the fundamental constants (see e.g. reviews [11, 12]).

The dependence of atomic transition frequencies on $\alpha$ may be presented in the following form

$$
\omega=\omega_{\mathrm{lab}}+q x, \quad x \equiv\left(\alpha / \alpha_{\mathrm{lab}}\right)^{2}-1 .
$$

In [13, 14], it was proposed to use transitions with significantly different $q$ factors for astrophysical and laboratory measurements of $\alpha$ variation. One can search for a variation of $\alpha$ by comparing two frequencies of atomic transitions over a long period of time. Following the Ref. [15] we can represent a measured quantity $\Delta(t)$ as

$$
\Delta(t)=\frac{d \ln \left(\omega_{1} / \omega_{2}\right)}{d t}=\left(\frac{\dot{\omega}_{1}}{\omega_{1}}-\frac{\dot{\omega}_{2}}{\omega_{2}}\right),
$$

where $\dot{\omega} \equiv d \omega / d t$. Taking into account Eq. (1) we can rewrite Eq. (2) as follows

$$
\Delta(t) \approx\left(\frac{2 q_{1}}{\omega_{1}}-\frac{2 q_{2}}{\omega_{2}}\right)\left(\frac{\dot{\alpha}}{\alpha_{\mathrm{lab}}}\right) .
$$

Narrow transitions with large and different $q$ values are of experimental interest.

Note that the atomic unit of energy cancels out in the ratio of two transition frequencies. The $\alpha$ dependence appears due to the relativistic corrections which rapidly increase with the nuclear charge $Z, \sim Z^{2} \alpha^{2}$, and strongly depend on the electron angular momentum. Therefore, transitions in heavy atoms with larger electron angular momentum difference (like $\Delta l=2$ for $s$ and $d$ orbitals) have larger $q$-coefficients. At present the best laboratory constraint on the temporal variation of $\alpha$ of $\dot{\alpha} / \alpha=(-1.6 \pm 2.3) \times 10^{-17} \mathrm{yr}^{-1}$ was obtained by Rosenband et al. in Ref. [16] by comparing the frequencies of the the ${ }^{2} S_{1 / 2} \rightarrow{ }^{2} D_{5 / 2}$ transition in ${ }^{199} \mathrm{Hg}^{+}$ $\left(q=-52200 \mathrm{~cm}^{-1}\right)$ and ${ }^{1} S_{0} \rightarrow{ }^{3} P_{0}^{o}$ transition in ${ }^{27} \mathrm{Al}^{+}$ $\left(q=146 \mathrm{~cm}^{-1}\right)$.

The relativistic corrections also rapidly increase with the effective charge $Z_{\text {eff }}$ which an external electron "sees". These corrections are proportional to $Z_{\mathrm{eff}}^{2}$. In the case of one electron above closed shells $Z_{\text {eff }}=Z_{i}+1$ where $Z_{i}$ is the ion charge. Therefore, highly charged ions are expected to have larger $q$. Unfortunately, the interval between the energy levels also increases $\sim Z_{\text {eff }}^{2}$, therefore, there is a risk to be out of the laser range. However, the Coulomb degeneracy and configuration crossing phenomena may help here. Indeed, in a neutral atom an electron energy level with larger orbital angular momentum is significantly higher than a level with lower orbital angular momentum with the same principal quantum number $n$.

For example, in the neutral Th the $5 s$ electron is a core electron while the $5 f$ electron is a valence electron. Respectively, the one-electron energy of the $5 f$ electron is much higher than that of the $5 s$ electron. Moreover, it is even higher than the energy of the $7 \mathrm{~s}$ electron. As seen from the experimental spectrum of the energy levels of the neutral (four-valence) Th [17], the energy of the $6 d^{2} 7 s 5 f$ state is higher than the energy of the $6 d^{2} 7 s^{2}$ 
state. In the hydrogen-like Th the energy of the $5 f$ state is equal to the energy of the $5 s$ state, i.e. it is significantly lower than the energy of the $7 s$ state. Therefore, there should be an ion charge at which the level $5 f$ "crosses" the level $7 s$ (at some higher charge it crosses $6 s$, etc). This kind of an approximate crossing (between the $5 f 6 d$ and the $6 d 7 s$ states) happens in $\mathrm{Th}^{2+}$, where the interval between them is about $5500 \mathrm{~cm}^{-1}$ only. The interval between the $5 f$ and $7 s$ states in $\mathrm{Th}^{3+}$ is also relatively small. Finally, the lifetime of the excited state should be large to have a narrow line.

All these requirements clearly point towards the $5 f_{5 / 2}-7 s_{1 / 2}\left(23131 \mathrm{~cm}^{-1}\right)$ transition in $\mathrm{Th}^{3+}$. It is also very important that the laser cooling of the ${ }^{232} \mathrm{Th}^{3+}$ ion has recently been reported by Campbell et al. in their paper [18]. This was the first time when a multiply charged ion has been laser cooled.

According to our calculations presented below the $5 f_{5 / 2}-7 s_{1 / 2}$ transition is a negative shifter with $q=$ $-75300 \mathrm{~cm}^{-1}$. Another narrow line in the same ion is the positive shifter $5 f_{5 / 2}-5 f_{7 / 2}\left(4325 \mathrm{~cm}^{-1}\right)$ with $q=+2900$ $\mathrm{cm}^{-1}$, this line may be used as a reference. Comparison may also be made with a positive shifter in another element, where $q$ may exceed $30000 \mathrm{~cm}^{-1}$ (see the table of atomic clock transitions with $q$ coefficients in review [19] and recent work [20]).

\section{METHOD OF CALCULATION}

To find $q$ factors we need to solve the atomic relativistic eigenvalue problem for different values of $\alpha$ or, respectively, for different values of $x$ from Eq. (11). The value of $x$ was chosen to be equal to $|x|=1 / 8$. This is a convenient choice that allows us to neglect nonlinear corrections and, on the other hand, to make calculations numerically stable. Thus, we need to calculate atomic frequencies $\omega_{ \pm}$for two values $x= \pm 1 / 8$. The corresponding $q$ factor is given by

$$
q=4\left(\omega_{+}-\omega_{-}\right)
$$

Since $\mathrm{Th}^{3+}$ is a univalent ion we have carried out calculations of its energy levels in the frame of the DiracHartree-Fock (DHF) method combined with many-body perturbation theory (MBPT). The latter allows us to take into account correlations between the valence electron and the core electrons.

We start from solving the DHF equations in the $V^{N-1}$ approximation. On the first stage the electrons of the closed core were included in a self-consistency procedure and their orbitals were found. After that we constructed the valence orbitals for several low-lying states using the frozen-core DHF equations. The virtual orbitals were determined with the help of a recurrent procedure described in [21]. The one-electron basis set of the following size was constructed: $1-20 s, 2-20 p, 3-20 d, 4-25 f, 5-18 g$.
TABLE I: The low-lying energy levels $\left(\right.$ in $\mathrm{cm}^{-1}$ ) in the DHF and the $\mathrm{DHF}+\Sigma$ approximations are presented. The theoretical values are compared with the experimental data.

\begin{tabular}{lcccc}
\hline \hline \multicolumn{2}{c}{ DHF } & \multicolumn{2}{c}{ DHF $+\Sigma$} & Experiment $^{a}$ \\
\hline $6 d_{3 / 2}$ & - & $5 f_{5 / 2}{ }^{b}$ & - & - \\
$6 d_{5 / 2}$ & 4225 & $5 f_{7 / 2}$ & 4800 & 4325 \\
$5 f_{5 / 2}$ & 5190 & $6 d_{3 / 2}$ & 9003 & 9193 \\
$5 f_{7 / 2}$ & 8617 & $6 d_{5 / 2}$ & 14749 & 14486 \\
$7 s_{1 / 2}$ & 11519 & $7 s_{1 / 2}$ & 21371 & 23131 \\
$7 p_{1 / 2}$ & 46702 & $7 p_{1 / 2}$ & 59487 & 60239 \\
$7 p_{3 / 2}$ & 58225 & $7 p_{3 / 2}$ & 72690 & 73056 \\
$8 s_{1 / 2}$ & 102595 & $8 s_{1 / 2}$ & 120106 & 119622 \\
$7 d_{3 / 2}$ & 103148 & $7 d_{3 / 2}$ & 120844 & 119685 \\
$7 d_{5 / 2}$ & 104763 & $7 d_{5 / 2}$ & 122603 & 121427 \\
$6 f_{5 / 2}$ & 111874 & $6 f_{5 / 2}$ & 128763 & 127262 \\
$6 f_{7 / 2}$ & 112316 & $6 f_{7 / 2}$ & 129251 & 127815 \\
$8 p_{1 / 2}$ & 117185 & $8 p_{1 / 2}$ & 135165 & 134517 \\
$8 p_{3 / 2}$ & 122194 & $8 p_{3 / 2}$ & 140552 & 139871 \\
$9 s_{1 / 2}$ & 142328 & $9 s_{1 / 2}$ & 161485 & 160728 \\
\hline \hline
\end{tabular}

${ }^{a}$ Reference [17];

${ }^{b}$ The removal energy of the $5 f_{5 / 2}$ state was found to be equal to $0.9414 \mathrm{au}$ on the DHF stage and $1.0578 \mathrm{au}$ on the

$(\mathrm{DHF}+\Sigma)$ stage. The experimental value is $1.0588 \mathrm{au}$.

At the next stage we included core-valence correlations $(\Sigma)$ into consideration and the wave functions were determined by solving the equation

$$
H_{\text {eff }}\left(E_{n}\right)\left|\Psi_{n}\right\rangle=E_{n}\left|\Psi_{n}\right\rangle,
$$

with an effective Hamiltonian defined as

$$
H_{\mathrm{eff}}(E)=H_{\mathrm{FC}}+\Sigma(E),
$$

where $H_{\mathrm{FC}}$ is the frozen-core DHF Hamiltonian and the self-energy operator $\Sigma$ is the energy-dependent correction, involving core excitations. In the following we will refer to it as the $\mathrm{DHF}+\Sigma$ formalism.

\section{DISCUSSION AND RESULTS}

In Table \we list the low-lying energy levels and compare them with the experimental data. To stress an importance of accounting for the core-valence correlations we present in Table \ the results obtained on the stage of pure DHF approximation and in the frame of $\mathrm{DHF}+\Sigma$ formalism.

As seen from Table I on the DHF stage even the order of the low-lying levels is incorrect. For instance, the $6 d_{3 / 2}$ state lays deeper than the $5 f_{5 / 2}$ state. An agreement between theoretical and experimental energy levels is rather poor. An accounting for the core-valence correlations ( $\Sigma$ corrections) recovers the correct order of the states. Besides that the theoretical energy levels become much closer to the experimental values.

To find the $q$ factors of the excited states in respect to the ground state $5 f_{5 / 2}$ we need to carry out calculations of frequencies $\omega_{ \pm}$for two values $x= \pm 1 / 8$. These 
TABLE II: The values of the $q$ factors $\left(\right.$ in $\mathrm{cm}^{-1}$ ) found in the $\mathrm{DHF}+\Sigma$ approximations are presented.

\begin{tabular}{lc}
\hline \hline & $q$ \\
\hline $5 f_{5 / 2}$ & - \\
$5 f_{7 / 2}$ & 2900 \\
$6 d_{3 / 2}$ & -39000 \\
$6 d_{5 / 2}$ & -34300 \\
$7 s_{1 / 2}$ & -75300 \\
$7 p_{1 / 2}$ & -67000 \\
$7 p_{3 / 2}$ & -48900 \\
$8 s_{1 / 2}$ & -57500 \\
$7 d_{3 / 2}$ & -50600 \\
$7 d_{5 / 2}$ & -46900 \\
$6 f_{5 / 2}$ & -46100 \\
$6 f_{7 / 2}$ & -45500 \\
$8 p_{1 / 2}$ & -57300 \\
$8 p_{3 / 2}$ & -50000 \\
$9 s_{1 / 2}$ & -55100 \\
\hline \hline
\end{tabular}

calculations are similar to those carried out for the laboratory value of the fine structure constant $\alpha_{\text {lab }}$. For this reason we do not discuss them in detail. We only stress again that it is important to include the $\Sigma$ corrections for obtaining the correct values of the frequencies $\omega_{ \pm}$and, respectively, the correct values of the $q$ factors.

In Table I we present the $q$ factors of the excited states listed in Table 1 in respect to the ground state, obtained on the DHF $+\Sigma$ stage. As follows from Eq. (4) the accuracy of the $q$ factors corresponds to the accuracy of calculations of $\omega_{+}$and $\omega_{-}$. The spectrum of the energy levels of $\mathrm{Th}^{3+}$ is not too dense and we believe that the energy levels obtained at $\alpha_{+}$and $\alpha_{-}$are found with the same accuracy as the energy levels computed at $\alpha_{\text {lab. As }}$ seen from Table \$ the latter are reproduced within the $10 \%$ accuracy. Correspondingly, the accuracy of the $q$ factors can also be estimated at the level of $10 \%$.

As seen from Table $\llbracket$ almost all $q$ factors are negative. As expected, the largest $q$ factor in absolute value was found for the $5 f_{5 / 2}-7 s_{1 / 2}$ transition. The only positive $q$ factor was obtained for the $5 f_{5 / 2}-5 f_{7 / 2}$ transition.

The transition frequency between fine structure levels of one multiplet $\omega_{J, J-1}$ (where $J$ is the total angular momentum) in the first order in $(\alpha Z)^{2}$ is given by the well known Landé rule: $\omega_{J, J-1}=A J(\alpha Z)^{2}$. It directly leads to $q_{J, J-1}=\omega_{J, J-1}$ (see Eq. (1)).

A marked difference between $q\left(5 f_{5 / 2}-5 f_{7 / 2}\right)=2900$ $\mathrm{cm}^{-1}$ and $\omega\left(5 f_{5 / 2}-5 f_{7 / 2}\right)=4325 \mathrm{~cm}^{-1}$ demonstrates an importance of the second order relativistic corrections $\sim(\alpha Z)^{4}$ for Th. These corrections modify the expression for $\omega_{J, J-1}$ leading to (see, e.g., 22] )

$$
\omega_{J, J-1}=A J(\alpha Z)^{2}+\left(B_{J}-B_{J-1}\right)(\alpha Z)^{4},
$$

where $A$ and $B_{j}$ are certain coefficients and $B_{j}$ are not small in comparison with $A$.

Since Th is the heavy element with $Z=90$, the parameter $(\alpha Z)$ is not small for it. In particular, $(\alpha Z)^{4} \approx 0.2$ and the term $\sim(\alpha Z)^{4}$ gives a noticeable contribution to the $q\left(5 f_{5 / 2}-5 f_{7 / 2}\right)$.

To conclude, we have calculated the $q$ factors for a number of excited states in $\mathrm{Th}^{3+}$ in respect to the ground state $5 f_{5 / 2}$. Our calculations showed that the $q\left(5 f_{5 / 2}-7 s_{1 / 2}\right)$ is very large in absolute value and, respectively, the $5 f_{5 / 2}-7 s_{1 / 2}$ transition is very sensitive to the temporal variation of the fine structure constant. Since the $7 \mathrm{~s}$ state is a metastable state, this transition is convenient for an experimental laboratory search of $\alpha$ variation. Another transition $\left(5 f_{5 / 2}-5 f_{7 / 2}\right)$ can be used as a reference.

\section{ACKNOWLEDGMENTS}

This work was supported by the Australian Research Council and Marsden grant. The work of S.G.P was supported in part by the Russian Foundation for Basic Research under Grants No. 07-02-00210-a and No. 08-0200460-a.
[1] J-P. Uzan, Rev. Mod. Phys. 75, 403 (2003).

[2] V. V. Flambaum and E. V. Shuryak, AIP Conf. Proc. 995, 1 (2007); e-print arXiv:physics/0701220

[3] J. K. Webb, V. V. Flambaum, C. W. Churchill, M. J. Drinkwater, and J. D. Barrow, Phys. Rev. Lett. 82, 884 (1999).

[4] J. K. Webb, M. T. Murphy, V. V. Flambaum, V. A. Dzuba, J. D. Barrow, C. W. Churchill, J. X. Prochaska, and A. M. Wolfe, Phys. Rev. Lett. 87, 091301 (2001).

[5] M. T. Murphy, J. K. Webb, and V. V. Flambaum, Mon. Not. R. Astron. Soc. 345, 609 (2003).

[6] M. T. Murphy, J. K. Webb, and V. V. Flambaum, Phys. Rev. Lett. 99, 239001 (2007).

[7] M. T. Murphy, J. K. Webb, and V. V. Flambaum, Mon. Not. R. Astron. Soc. 384, 1053 (2008).
[8] E. Reinhold, R. Buning, U. Hollenstein, A. Ivanchik, P. Petitjean, and W. Ubachs, Phys. Rev. Lett. 96, 151101 (2006).

[9] V. F. Dmitriev, V. V. Flambaum, and J. K. Webb, Phys. Rev. D 69, 063506 (2004).

[10] V. V. Flambaum and R. B. Wiringa, Phys. Rev. C 76, 054002 (2007).

[11] V. V. Flambaum, Int. J. Mod. Phys. A 22, 4937 (2007).

[12] S. N. Lea, Rep. Prog. Phys. 70, 1473 (2007).

[13] V. A. Dzuba, V. V. Flambaum, and J. K. Webb, Phys. Rev. Lett. 82, 888 (1999).

[14] V. A. Dzuba, V. V. Flambaum, and J. K. Webb, Phys. Rev. A 59, 230 (1999).

[15] V. A. Dzuba, V. V. Flambaum, and M. V. Marchenko, Phys. Rev. A 68, 022506 (2003). 
[16] T. Rosenband, D. B. Hume, P. O. Schmidt, C. W. Chou, A. Brusch, L. Lorini, W. H. Oskay, R. E. Drullinger, T. M. Fortier, J. E. Stalnaker, et al., Science 319, 1808 (2008).

[17] NIST, Atomic Spectra Database, URL http://physics.nist.gov/cgi-bin/AtData/main_asd.

[18] C. J. Campbell, A. V. Steele, L. R. Churchill, M. V. DePalatis, D. E. Naylor, D. N. Matsukevich, A. Kuzmich, and M. S. Chapman, Phys. Rev. Lett. 102, 233004 (2009).
[19] V. V. Flambaum and V. A. Dzuba, Can. J. Phys. 87, 25 (2009), e-print arXiv:0805.0462.

[20] S. G. Porsev, V. V. Flambaum, and J. R. Torgerson, Phys. Rev. A 80, 042503 (2009).

[21] M. G. Kozlov, S. G. Porsev, and V. V. Flambaum, J. Phys. B 29, 689 (1996).

[22] M. G. Kozlov, S. G. Porsev, S. A. Levshakov, D. Reimers, and P. Molaro, Phys. Rev. A 77, 032119 (2008). 